\title{
O Luto em Adultos Idosos: Natureza do Desafio Individual e das Variáveis Contextuais em Diferentes Modelos
}

\author{
Bereavement in Older Adults: Individual Challenge \\ and Contextual Variables in Different Models
}

\author{
Maria das Dores Ferreira da Silva \& José Ferreira-Alves* \\ Universidade do Minho, Braga, Portugal
}

\begin{abstract}
Resumo
A perda por viuvez em idade avançada é, porventura, de entre vários acontecimentos do ciclo de vida, um dos mais normativos e, simultaneamente, dos menos investigados. Com o aumento da esperança de vida, a viuvez nos adultos idosos ocorre cada vez mais tarde no ciclo de vida, é um acontecimento expectável e parece haver vantagens em conceptualizá-lo como uma importante transição psico-social que implica a reconfiguração do significado da vida e dos seus propósitos. Neste artigo abordaremos alguns dos principais modelos do processo de luto e algumas variáveis individuais e contextuais que determinam reacções associadas à perda. Faremos uma particular focalização no modelo proposto por Stroebe e Schut bem como nas suas implicações teóricas e práticas.

Palavras-chave: Luto, adultos idosos, processo dual, viuvez.

Abstract

The loss of a spouse in old age is one of the most significant, but least investigated events in the life cycle. With increasing life expectancy, widowhood in older adults occurs increasingly later in the life cycle. It is an expectable event and may be advantageously conceptualized as a major psychosocial transition that involves the reconfiguration of the meaning of life and its purpose. The present article discusses some of the main models of the grieving process and the effect of some individual and contextual variables on reactions regarding the loss of a spouse. It focuses mainly on Stroebe and Schut dual process model as well as on its theoretical and practical implications.

Keywords: Bereavement, older adults, dual process, widowhood.
\end{abstract}

A perda por viuvez em idade avançada é, porventura, de entre vários acontecimentos do ciclo de vida, um dos mais normativos e, simultaneamente, dos menos investigados. Além dos desafios inerentes à falta de projectos ou expectativas culturais para a idade avançada, a perda por viuvez aparentemente adiciona complexidade e dificuldade à vida do adulto idoso, pelos novos problemas que deve enfrentar e lidar. A viuvez é uma ocorrência maioritariamente feminina, tendência que tende a acentuar-se perante dados demográficos que revelam uma maior capacidade das mulheres em sobreviverem aos seus maridos. Com o aumento da esperança de vida, a viuvez nos adultos idosos ocorre cada vez mais tarde no ciclo de vida, é um acontecimento expectável e parece haver vantagens em conceptualizá-lo como uma importante transição psico-social que implica a reconfiguração do significado da vida e dos seus propósitos (Parkes, 1988, 1993).

\footnotetext{
* Endereço para correspondência: Departamento de Psicologia Básica, Universidade do Minho, Campus de Gualtar, Braga, Portugal 4710-057. E-mail: alves@psi.uminho.pt
}

Para os adultos idosos a viuvez, para além de ser um acontecimento com um grau elevado de probabilidade de acontecer, quando comparado com a população mais jovem (Bennet, 1997; S. M. Moss, Moss, \& Hansson, 2001; Schaefer \& Moos, 2001), constitui uma ocorrência central da idade avançada, pelas mudanças pessoais, familiares e sociais que implica - para além da sua influência no bem-estar físico e psicológico da população enlutada (Bennet, 2005; Carr \& Bordnar-Deren, 2009; Lee \& Carr, 2007; Ward, Mathias, \& Hitchings, 2007). Ao mesmo tempo, a viuvez em idade avançada, tal como em outras idades, tem um importante impacto na identidade e no sentido da própria vida, que desafia o aparecimento de novas orientações para significar a perda e, também, implica novas exigências práticas do viver diário. Num recente estudo realizado em lares residenciais na segunda cidade portuguesa, as perdas que apresentavam as consequências físicas, emocionais, cognitivas e comportamentais mais negativas foram, em primeiro lugar, a do cônjuge e depois, já bem distante, as perdas dos irmãos e dos amigos (Malheiros \& Ferreira-Alves, 2010). A transição que o luto representa tem sido conceptualizada teorica- 
mente de forma plural, após um período de cerca de um século onde a perda era vista, mesmo na ausência de suporte empírico, como um período que exigia um trabalho de luto cujo fim último era apenas o desligamento do objecto da perda (Bonanno \& Kaltman, 1999).

Nesta revisão selectiva de investigação, abordaremos de forma sumária alguns dos principais modelos teóricos dos processos de luto e algumas variáveis individuais e contextuais que determinam as reacções e os comportamentos associados à perda. Mostraremos como é tão importante conhecer os diferentes modelos de entendimento do processamento do luto, como as variáveis que, em vários estudos empíricos, se revelaram factores facilitadores ou problemáticos do seu curso. Enfatizaremos o Processo Dual de Lidar com o Luto, proposto por Stroebe e Schut (1999), que nos apresenta como tarefas fundamentais do luto, o confronto e o evitamento de estressores orientados para a perda e para o restabelecimento.

\section{Modelos Teóricos}

De entre os vários modelos e teorias explicativas do processo de luto, aqueles que a seguir passamos a descrever parecem-nos centrais numa abordagem histórica e são considerados fundamentais no entendimento do luto.

\section{Teoria do Trabalho de Luto (Grief Work)}

As origens do conceito "trabalho de luto" podem ser encontradas em Freud, mais precisamente no seu artigo "Mourning and Melancholia", escrito em 1915 e publicado em 1917. Este conceito serviu de fundamento a muita literatura sobre a dor e o luto, sendo este definido por Freud (1917/2001), como um processo de diminuição gradual de energia, que liga o indivíduo enlutado ao objecto perdido ou à pessoa falecida. Durante este processo, a pessoa enlutada tem que enfrentar a realidade da perda e começar a desvincular-se da pessoa falecida. De resto era esta a principal tarefa do trabalho de luto - quebrar os laços com a pessoa falecida. Trabalhar o luto, nesse sentido, significaria confrontar as emoções e os sentimentos associados à perda, que incluem falar sobre aspectos relacionados com o morto (chorar a morte, expressar tristeza ou saudades do morto) e as circunstâncias da morte. A expressão de sentimentos e, em geral, o falar sobre o ente querido desaparecido, são o que se chama o "trabalho do luto" que levará à perda gradual dos laços com a pessoa falecida (memórias, por exemplo). Caso este objectivo não se concretize então, segundo Freud (1917/ 2001), estaremos na presença de um luto patológico. Antes, porém, há uma tentativa inicial de manter os laços com a pessoa falecida. $\mathrm{O}$ enlutado, incapaz de abandonar o objecto perdido, testa a realidade da perda através da inclusão da pessoa falecida na sua vida. A pessoa enlutada toma consciência da inviabilidade destas tentativas $\mathrm{e}$, através do teste da realidade (tomando consciência da realidade da perda), chega à conclusão que manter os laços com o morto é impossível. O indivíduo fica, então, numa situação de desapego da pessoa morta, adquirindo liberdade para investir e reorientar as suas emoções e a sua atenção a outras coisas, nomeadamente a outras relações e a novas relações (Russac, Steighner, \& Canto, 2002).

\section{Teoria da Vinculação}

Bowlby (1980) sustenta que os tipos de vinculação (seguro ou inseguro), experienciados por uma pessoa na sua família de origem se associam a diferentes formas e resultados de reacção à perda. Assim é, porque os tipos e os comportamentos de vinculação caracterizam, também, alguns aspectos da natureza dos laços entre pessoas adultas e parecem não ser independentes dos laços ou vinculação estabelecida durante a infância. A teoria da vinculação de John Bowlby foi - e continua a ser - integrada em diversas pesquisas e investigações associadas à perda, sendo pioneira na introdução do conceito de vinculação como um conceito central para entender não só as ligações humanas mas, também, as reacções à perda dessas ligações (Canavarro, 2004). Bowlby (1980) caracteriza o processo de luto através de quatro fases: fase de entorpecimento, fase de saudade e de procura da pessoa falecida, fase de desorganização e desespero e fase de reorganização. Repare-se na diferença entre a ideia de desvinculação - seria o objectivo de um luto para Freud - e a ideia de reorganização, como condições de um processo de luto bem-sucedido. A teoria da vinculação é, fundamentalmente, uma teoria de relações, de ligações, de laços que existem entre pessoas, desde a díade criança-cuidador, passando pelas relações românticas (Hazan \& Shaver, 1987) até às reacções demonstradas pela morte do cônjuge, por exemplo. Constitui, por isso, uma excelente ferramenta para o entendimento de diferenças individuais no luto.

\section{Tarefas de Worden}

Worden (2002) define um modelo baseado em tarefas que a pessoa enlutada deve desempenhar para se adaptar ao luto. Este autor considera que este conceito de tarefas se identifica mais com o conceito de trabalho de luto de Freud implicando, da parte do enlutado, uma tomada de acção no processo de luto e a consciência de que está ao seu alcance fazer alguma coisa para o resolver. As tarefas definidas por Worden (2002) são quatro: (a) aceitar a realidade da perda, (b) trabalhar a dor do luto, (c) adaptarse a um ambiente no qual a pessoa falecida está ausente, (d) recolocar, emocionalmente, a pessoa falecida e continuar a viver.

\section{As Etapas de Kübler-Ross}

Kübler-Ross (1997) através dos métodos que usou para estudar a perda - centrados na pessoa - tornou o luto um assunto clínico, educativo e de investigação. A clareza com que mostrou compreender casos de luto, não se distanciando da linguagem dos próprios enlutados, popularizou o seu modelo de etapas. Esta autora definiu cinco etapas inicialmente referidas à experiência típica de um 
grupo de doentes em fase terminal, mas que foram, também, observadas nos familiares desses doentes. Desta forma, as etapas (negação, raiva, negociação, depressão e aceitação), definidas por Kübler-Ross, passaram a ser um instrumento de compreensão e de gestão de situações de luto. Note-se que as etapas propostas por Kübler-Ross (1997) devem ser entendidas como atribuindo um papel activo ao indivíduo; não são simplesmente fases pelas quais naturalmente e passivamente as pessoas passam, mas antes, manifestações de uma adaptação activa ao confronto com a perda.

Os modelos que acabamos de sumariar constituem propostas de grande relevo para a compreensão da forma como o luto se desenrola. Contudo, essas propostas não esgotam as possibilidades de novas construções. Daremos um privilégio especial nesta revisão à proposta de Stroebe e Schut (1999).

\section{Processo Dual de Lidar com o Luto}

Stroebe e Schut (1999), propuseram um modelo dual de compreensão das reacções dos indivíduos às perdas e de forma geral sugerem um modelo de compreensão do processo de "coping" e de adaptação ao luto. No modelo do processo dual de lidar com o luto (Dual Process Model of Coping with Bereavement [DPM]), proposto por Stroebe \& Schut, 1999), coexistem três dimensões ou componentes: (a) orientação para a perda, (b) orientação para o restabelecimento e (c) oscilação. Cada uma destas orientações reflecte a resposta a estressores que emergem com a perda: estressores da própria perda, da quebra dos laços com o ente querido, por um lado e, por outro lado, estressores ligados ao restabelecimento, à resposta aos desafios para prosseguir a sua vida individual sem o ente querido. Cada indivíduo pode escolher confrontar ou evitar esses estressores de perda e de restabelecimento, o que leva a que seja interessante e heurística a proposta do conceito de oscilação. Na orientação para a perda a pessoa enlutada centra a sua atenção em aspectos da perda relacionados com a pessoa falecida: laços afectivos (necessidade de recolocação dos laços), relação conjugal, chorando, focando-se nas circunstâncias da morte (Hansson \& Stroebe, 2007; Stroebe \& Schut, 1999). A orientação para o restabelecimento refere-se a consequências secundárias à perda que constituem fontes de stress com as quais a pessoa enlutada necessita de lidar, bem como à definição de formas de como o fazer (Hansson \& Stroebe, 2007). São exemplos os problemas financeiros, agora que o cônjuge faleceu, o estatuto de viúva, tratar de assuntos que antes eram da responsabilidade do cônjuge falecido. Todos estes aspectos vão tornar o "fardo" da perda mais pesado, obrigando a viúva a definir para estes estressores estratégias de "coping" adequadas. A oscilação surge como a dimensão mais distintiva deste modelo relativamente aos outros modelos e teorias: "O DPM é claramente distinto de todos os outros modelos de coping com o luto devido a um processo de regulação emocional envolvendo uma oscilação" (Hansson \& Stroebe, 2007, p. 48). A oscilação refere-se à alternância entre a orientação para a perda e a orientação para o restabelecimento realizada através do confronto e do evitamento dos diferentes estressores do luto. Num momento, a pessoa enlutada confrontará algum aspecto da perda; noutro, contudo, evitará memórias, procurará distrair-se, ver um filme, sair com amigos e, deste modo, não lidar com qualquer aspecto da perda. Haverá momentos, no entanto, em que se tornará necessário confrontar aspectos secundários à perda, como executar tarefas que antes eram da responsabilidade do falecido marido (Hansson \& Stroebe, 2007). Atender em simultâneo às duas dimensões é virtualmente difícil ou impossível; mas como é preciso atender às duas dimensões, a oscilação é um processo necessário e muito possivelmente um dos melhores indicadores da evolução da experiência do luto (Figura1).

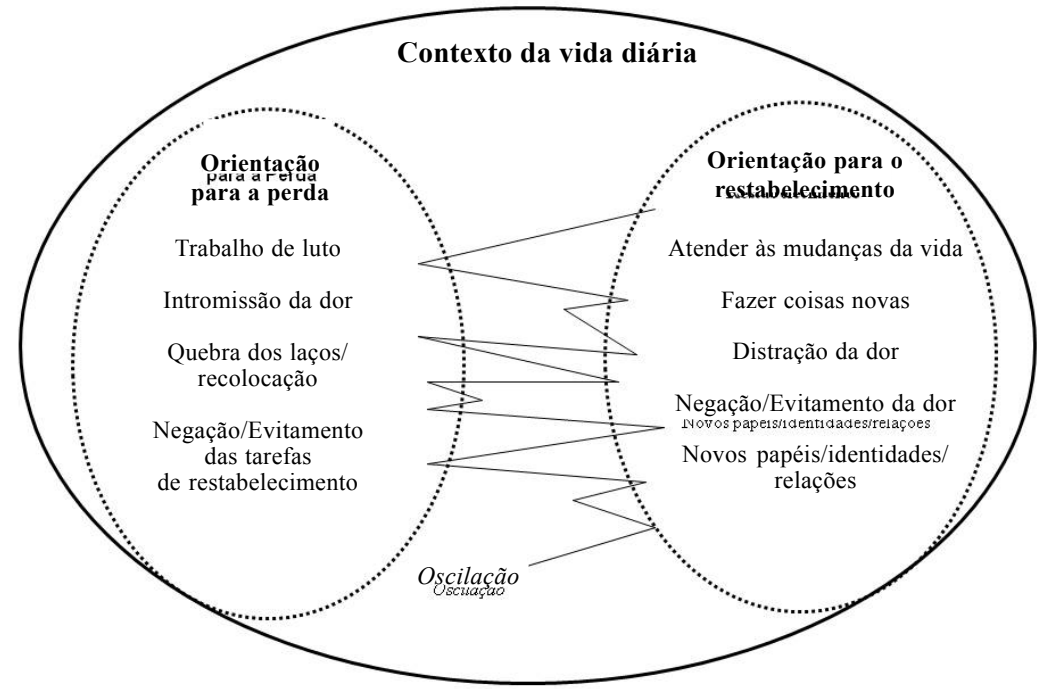

Figura 1. Processo Dual de Lidar com o Luto (Hansson \& Stroebe, 2007)1. 
Resumindo, neste modelo, aquilo que é usualmente considerado o objecto principal da perda e o seu stressor principal - o desaparecimento ou falecimento de uma pessoa, por exemplo - é visto apenas como uma parte do processo de luto. A outra parte prende-se com a experiência de estressores secundários à perda, isto é, estressores de restabelecimento, de prosseguimento da vida de uma forma diferente da anterior e que necessitam, tal como os estressores relacionados com a perda, de ser confrontados e/ou evitados. Por outro lado, o confronto e o evitamento de ambos os tipos de estressores resultam num processo a que Stroebe e Schut (1999) chamaram de "oscilação" que poderá ser um bom indicador da evolução de um processo de luto. O valor que os autores emprestam ao processo de oscilação como um processo de "coping" que regula os esforços de adaptação do indivíduo, em paralelo com a sua proposta de que um "coping" adaptativo seria constituído tanto por estressores de perda como de restabelecimento é a assunção de que a oscilação é um indicador do processo.

Esta breve compilação das diversas perspectivas de processamento da perda mostra-nos que os contributos de cada uma são válidos e relevantes no entendimento do processo. Trabalhar o luto continua a ser necessário; o tipo de vinculação desenvolvido com a pessoa falecida constitui uma variável determinante do processo; o indivíduo enlutado deve aceitar a realidade da perda e reorganizar a sua vida sem a pessoa falecida. Stroebe e Schut (1999) elaboraram o seu próprio modelo do luto, integrando elementos das restantes teorias. Fará todo o sentido que se tenha em conta esta dinâmica, imprimida pelo movimento de oscilação, ao longo do tempo e integrá-la em estudos que avaliem os efeitos de variáveis individuais e contextuais no processamento do luto, permitindo-nos uma melhor compreensão do fenómeno que é o luto (Rijo, 2004).

\section{Variáveis Individuais e Contextuais}

Um dos traços característicos dos modelos teóricos anteriormente revistos é que não teorizam, nem deles se conhecem dados empíricos, acerca do luto numa perspectiva de ciclo de vida. Significa que parecem ter olhado para o luto a partir de uma pessoa prototípica que dificilmente representa com precisão a experiência de pessoas em diferentes momentos do ciclo de vida. Na revisão que se segue adicionamos informação e clarificação da experiência do luto apenas em idade avançada.

As consequências causadas pela viuvez são muito abrangentes e podem depender de muitos factores, como as circunstâncias da morte, a idade, o género, a qualidade da relação conjugal, a personalidade do enlutado e da pessoa falecida, o tipo de perda (cônjuge, filho, pai...), a qualidade da morte, a ausência de apoio social, entre outros (Parkes, 1988; Sanders, 1993; Worden, 2002). Centraremos a nossa atenção em três variáveis que diferente literatura tem considerado relevante na adaptação ao luto e no entendimento das reacções individuais à perda: qualidade da relação conjugal, circunstâncias da morte e o género (Carr \& Utz, 2002; Hansson \& Stroebe, 2007; Johnson, Vanderwerker, Bornstein, Zhang, \& Prigerson, 2006). Este conhecimento permitir-nos-á compreender como estas variáveis podem estar associadas a diferentes experiências do luto (com diferentes graus de sucesso) vividas pelos indivíduos enlutados. Abordaremos o impacto destes factores nas reacções de adultos idosos viúvos.

Algumas das investigações que a seguir apresentamos baseiam-se em dados fornecidos por um estudo realizado nos Estados Unidos ([CLOC] Changing Lives of Older Couples) entre Junho de 1987 e 1993. Em termos gerais este estudo envolveu adultos idosos (homens e mulheres) com 65 e mais anos de idade e pretendia saber mais acerca do luto conjugal nesta população. Os dados foram obtidos prospectivamente e longitudinalmente ao longo de 6, 18 e 48 meses após a perda e a amostra foi entrevistada antes e depois da perda (Carr \& Utz, 2002; Carr et al. 2000; Carr, House, Wortman, Nesse, \& Kessler, 2001). Um grupo de controlo também fez parte do estudo. O número de participantes e a duração do estudo $(6,18$ ou 48 meses) foram definidos conforme os objectivos de cada investigação. Estas características fazem com que a partir desta pesquisa sejam publicados vários artigos especialmente interessantes sobre o luto (Hansson \& Stroebe, 2007).

\section{Qualidade da Relação Conjugal}

Tal como Bradley e Cafferty (2001) afirmam, a relação conjugal nos adultos idosos é particularmente importante, porque as pessoas se confrontam com a possibilidade de não terem outro tipo de relação próxima. O estudo do impacto da qualidade da relação conjugal no processo do luto, contribuiu para um melhor entendimento do quanto o luto pode ser um processo heterogéneo e singular. Carr et al. (2000) levaram a cabo uma investigação longitudinal, baseada no CLOC, cujo principal objectivo era o de saber se a adaptação psicológica é influenciada por três aspectos da qualidade da relação conjugal: proximidade, conflito e dependência instrumental. A amostra deste estudo envolveu a participação de 203 pessoas viúvas (53 homens e 150 mulheres) e um grupo de controlo de indivíduos casados. Os participantes tinham 65 e mais anos de idade e eram não institucionalizados. A recolha de dados foi feita através de entrevistas efectuadas 6 meses após a perda. Os resultados mostraram o seguinte: quando comparados com o grupo de controlo, o grupo de adultos idosos viúvos revela que a viuvez constitui um importante preditor da depressão, não diminuindo quando são introduzidos na análise os indicadores da qualidade da relação conjugal. Por outro lado, como seria de esperar, os adultos idosos que relataram elevada dependência instrumental do cônjuge apresentaram, com a morte deste, valores de ansiedade mais elevados se comparados com aqueles que tinham menos dependência. Esta investigação acabou por dar suporte à ideia de que a dependência 
instrumental do cônjuge (no desempenho de tarefas como, por exemplo, reparações em casa ou gerir finanças) constitui um forte preditor de ansiedade para as mulheres viúvas. A adaptação à viuvez parece apresentar mais dificuldades não só para aqueles com elevados níveis de proximidade e dependência instrumental do cônjuge falecido, mas também para aqueles com baixos níveis de conflitos no casamento. Já os que viveram um casamento conflituoso apresentaram baixos níveis de saudade nos primeiros 6 meses após a perda, em contraste com as pessoas que se relacionavam de forma próxima e com grande interdependência (Carr et al., 2000).

Numa outra investigação longitudinal Lund, Caserta e Dimond (1993), em colaboração com outros colegas, pretenderam descrever o processo de adaptação à morte do cônjuge, examinar os factores que influenciam as consequências e identificar possíveis formas e estratégias para intervenção. A amostra era constituída por 192 cônjuges (homens e mulheres) enlutados recentemente (por viuvez) e um grupo de controlo com 104 indivíduos casados com 50 e mais anos de idade. As características desta amostra envolveram indivíduos com idades compreendidas entre os 50 e os 93 anos de idade e uma média de idades de 67,6 anos $(D P=8,2)$. Os participantes foram contactados uma primeira vez cerca de 3 semanas após a perda. Os restantes contactos efectuaram-se 2 meses, 6 meses, 1 ano, 18 meses e 2 anos após a perda. Os instrumentos utilizados nestes 6 contactos foram questionários e entrevistas idênticos, quer para o grupo de adultos idosos enlutados, quer para o grupo de controlo. A maioria era do sexo feminino, $74 \%$ do total da amostra de 192 adultos idosos enlutados. Alguns dos resultados deste estudo mostraram que, durante o processo de adaptação à perda, a falta de habilidades para lidar com problemas da vida diária, surge como uma dificuldade associada ao processo. Estas, são diferentes para homens e mulheres enlutados, sendo que os homens são mais deficitários em capacidades relacionadas com a cozinha, as compras e a limpeza da casa (tarefas tradicionalmente femininas) e as mulheres mais deficitárias em capacidades relacionadas com as reparações da casa, em gerir finanças e questões legais (tarefas tradicionalmente masculinas). Lund et al. (1993), revelaram que quanto maior for o nível de competências do cônjuge enlutado, maior será a capacidade em gerir e exercer as tarefas do dia-a-dia conduzindo a uma adaptação mais bem-sucedida do luto. Estes dados remetem-nos para o possível impacto da interdependência conjugal na realização de determinadas tarefas e, por conseguinte, da qualidade da relação conjugal como um factor determinante no desenrolar do processo do luto. Estas conclusões vão de encontro aos resultados da investigação de Carr et al. (2000). Um outro resultado encontrado por Lund et al. (1993) refere-se à identificação da solidão como a maior dificuldade reportada pelos idosos enlutados, persistindo ao longo dos 2 anos. Relativamente a outros problemas associados ao luto, Lund et al. (1993) encontram os seguintes: depressão, relações fami- liares, amizades, saúde física, identidade pessoal e viver de acordo com as próprias expectativas e com as expectativas de outros. Verificaram que os valores da maior parte destes problemas melhoraram ao longo do período de 2 anos. Em busca dos melhores preditores do processo de luto Lund et al. (1993) encontraram a auto-estima e as competências pessoais no desempenho das tarefas diárias como os factores que mais influenciaram o bemestar dos adultos idosos, o qual apresentava pontuações mais positivas em cada um dos momentos subsequentes de recolha de dados. Observou, ainda, que estes dois preditores se relacionavam entre si. Os indivíduos que apresentavam um nível de competências elevado apresentavam, igualmente, níveis de auto-estima elevados. Em 2004 um estudo de Carr, baseado nos dados do CLOC envolvendo adultos idosos com 65 e mais anos de idade, mostrou que as mulheres mais dependentes dos maridos apresentavam o nível mais baixo de auto-estima enquanto casadas. Contudo, depois de sofrerem a perda do cônjuge o nível de auto-estima aumentou. Este dado é curioso, mostrando manifestamente que a qualidade da relação se relaciona de facto não só com as reacções à morte mas pode ter também impactos vários na mudança pessoal. De uma maneira geral, os resultados destes estudos traduzem, além das complexidades da relação conjugal, uma visão tradicional de divisão e desempenho de papéis durante o casamento e baseados no género, reflectindo-se, posteriormente na vivência do luto.

\section{Circunstâncias da Morte}

As circunstâncias em que a morte ocorreu, isto é, se a morte ocorreu de uma forma repentina e súbita ou se era esperada, constituem um stressor cujo impacto se faz sentir no comportamento das pessoas enlutadas. A chegada esperada de uma morte pode trazer alguma vantagem à pessoa enlutada, na medida em que permite a esta conversar com a pessoa que se encontra em vias de falecer e, deste modo, ir trabalhando o seu luto (Schaefer \& Moos, 2001), levando-nos a pensar que perder o cônjuge é mais um processo que um acontecimento. Cavanaugh (1993) afirma ainda não estarem bem entendidas as razões pelas quais as mortes antecipadas conduzem a uma recuperação mais rápida. Já a morte repentina surge como factor de risco do processo de luto, contribuindo para o aparecimento de um luto complicado (Parkes, 1998; Sanders, 1993).

Carr et al. (2001) realizaram uma investigação baseada no CLOC que teve como objectivos: identificar a relação entre a morte antecipada e a adaptação psicológica (medida através da depressão, ansiedade, saudade, choque, raiva, pensamentos intrusivos) de adultos idosos 6 e 18 meses após a perda, ou seja, saber que dimensões da adaptação psicológica são afectadas por uma morte antecipada; avaliar como é que a relação entre a morte antecipada ou esperada e o bem-estar das pessoas viúvas difere entre homens e mulheres; avaliar como é que o efeito da morte antecipada é mediado ou anulado pelas características 
do contexto em que a morte ocorreu (cuidados prestados antes da morte, cuidados de enfermagem em casa, idade do cônjuge enlutado e a comunicação entre o casal antes da morte). O total de participantes envolveu cerca de 365 adultos idosos (261 mulheres e 104 homens). Os resultados obtidos foram os seguintes: a morte esperada não está relacionada com a depressão, a dor, a raiva e o choque nos 6 e 18 meses após a perda; as mortes súbitas estão relacionadas com níveis elevados de pensamentos intrusivos (dificuldade em adormecer, pensamentos constantes sobre a pessoa falecida, tentativa de bloquear memórias sobre a pessoa falecida) seis meses após a perda, mas com uma diminuição a partir do décimo oitavo mês; os níveis de ansiedade aumentam, seis e dezoito meses após a perda, quando a morte é esperada e a espera prolongada. O impacto desta variável, morte esperada ou repentina, parece provocar padrões diferentes de resposta, mas não de forma linear, pois há outras variáveis como o tempo de espera que podem ter um significativo impacto no processo de luto. Este estudo reflecte a importância que deve ser dada ao contexto em que a morte ocorre. Por exemplo, os idosos enlutados que tiveram oportunidade de discutir a morte com o seu cônjuge e aqueles cujos cônjuges residiram em instituições, apresentaram baixos níveis de pensamentos intrusivos e níveis significativamente mais baixos de ansiedade nos dois momentos das entrevistas (6 e 18 meses após a perda); já a comunicação entre o cônjuge falecido e o cônjuge sobrevivente não influenciou os níveis de ansiedade nos 6 e 18 meses após a perda; de todas as variáveis que compõem o contexto da morte, somente uma revelou alguma significância no comportamento da saudade: o fornecimento de cuidados ao cônjuge antes da morte foi associado a um aumento da saudade 6 meses após a perda, diminuindo com o passar do tempo. Os homens, cujas esposas faleceram repentinamente, continuaram a mostrar níveis mais baixos de saudade, 18 meses após a perda, que aqueles cuja morte das esposas já era esperada; este facto obteve um resultado oposto no respeitante às mulheres.

\section{Género}

Uma revisão da literatura mostrou que apesar de viúvos e viúvas sofrerem com a experiência do luto, os viúvos apresentam consequências mais severas que as viúvas (Stroebe, 1998). Tal como Stroebe, Bennet (1997) reviu investigação relacionada com os efeitos da viuvez em mulheres adultas idosas a médio e a longo prazo. Apesar de ter constatado que os estudos longitudinais sobre os efeitos da viuvez em idade avançada não são muitos, esta autora concluiu que os efeitos produzidos pela perda, sobre a saúde mental, continuaram a fazer-se sentir por vários anos após a perda. Foram observados níveis elevados de depressão e uma saúde mental mais pobre. Verificou-se que este estado depressivo diminuía ao longo dos anos, ao contrário da moral que aumentava. A participação social e o convívio permaneceram inalteráveis no médio prazo, tal como a saúde física. Num outro estudo, semelhante ao anterior, realizado por Bennet (1998) em relação a homens viúvos, os resultados mostraram, também, haver uma diminuição na saúde mental dos homens e um aumento da depressão, que esteve associado a uma diminuição da sua participação social, em contraste com a participação social das mulheres que não foi alterada, mas que a longo prazo diminuiu, só como função da idade e não da viuvez (Bennet, 1997). Parece, pois, que ao longo do tempo, a depressão, como consequência da viuvez, acarreta consequências mais negativas para os homens do que para as mulheres. Um outro estudo, realizado por van Grootheest, Beekman, Broese van Groenou e Deeg (1999), com uma amostra de adultos idosos com idades entre os 55 e os 85 anos $(M=70,8$ e $D P=8,7$ para os homens e $M=70,5$ e $D P=8,8$ para as mulheres) e adultos idosos casados (grupo de controlo), pretendeu averiguar a influência da viuvez nas taxas de depressão entre homens e mulheres enviuvados, ao longo do tempo, bem como se o género, o tempo de viuvez, o suporte social, preocupações financeiras ou domésticas poderiam explicar essa relação. Os resultados apontaram para 3 conclusões:

1. A intersecção do género com o estatuto conjugal só explicava os sintomas depressivos se a viuvez tivesse ocorrido há mais de 4 anos em desfavor dos homens viúvos.

2. A intersecção do género e do estatuto conjugal explicava (a) o suporte instrumental recebido em favor das mulheres viúvas, (b) a menor satisfação com o vencimento das mulheres viúvas e (c) a maior realização de tarefas domésticas leves pelos homens viúvos e uma menor realização destas tarefas leves pelas mulheres; finalmente,

3. Confirmou-se a hipótese já veiculada por estudos prévios de que a viuvez está positivamente associada à depressão; mas também se verificou uma capacidade preditiva das variáveis "estado funcional" e "número de doenças crónicas" em predizer positivamente os valores da depressão de pessoas viúvas de ambos os sexos; o tamanho da rede social e a satisfação com o vencimento auferido predizem de forma inversa os valores da depressão; adicionalmente, há variáveis, nomeadamente o suporte instrumental e emocional recebido que predizem, respectivamente, de forma directa e inversa, os valores da depressão nos homens viúvos; por seu lado a ajuda nas tarefas domésticas prediz positivamente os valores da depressão nas mulheres viúvas. Por último, as variáveis "trabalhos domésticos leves" e trabalhos domésticos pesados" bem como a "idade" não apresentaram qualquer poder preditivo dos valores da depressão.

Numa pesquisa realizada por Peggy e Kalyani (2001) sobre a forma como as viúvas em Singapura experienciam a sua viuvez, os resultados aparentemente reflectem algum impacto da divisão de papéis numa relação conjugal. Peggy e Kalyani (2001) constataram que 10,3\% 
dos viúvos estavam preocupados com as tarefas diárias, enquanto só $7,3 \%$ das viúvas apresentavam essa preocupação. Em relação às questões financeiras, verificou-se o contrário, ou seja $24,7 \%$ das viúvas contra $16,2 \%$ de viúvos. Carr e Utz (2002) consideram, a este propósito, que para os homens e mulheres socializados com um casamento tradicional em que predominam os papéis tradicionais associados ao género, a viuvez pode representar para os homens, a perda de uma gerente da casa, de uma confidente e de alguém que tomava as decisões; para as mulheres, a viuvez poderá tendencialmente representar mais a perda de um recurso financeiro. Esta divisão tradicional de papéis coloca, pois, diferentes desafios para viúvos e viúvas. Como será, então, que viúvos e viúvas reorganizam a sua vida após a morte do cônjuge? Carr (2006) revela-nos que os viúvos se sentem mais motivados para voltar a casar que as viúvas, procurando novas companheiras logo após a morte do cônjuge. Esta atitude constitui uma forma de recuperarem da solidão e da tristeza provocadas pela perda. Já as viúvas preenchem o vazio emocional deixado pela perda, estabelecendo relações com amigos, crianças, familiares ou participando em actividades diversas. Num estudo efectuado com viúvas e viúvos idosos, Davison (2002) afirma que as mulheres não desejam voltar a casar especialmente por uma ou outra das três razões seguintes: não desejam cuidar de outro homem, tiveram um casamento feliz e o seu marido não podia ser substituído. Já os homens revelaram um desejo maior de voltar a estabelecer uma relação romântica, de preferência com mulheres mais novas. Contudo, esta decisão dependia da idade e da sua saúde: quanto mais frágil e mais velho o viúvo fosse, menos probabilidade havia de estabelecer novas relações.

\section{Considerações Finais}

Para melhor compreendermos as diferenças individuais no processamento da perda é necessário compreender como estes processos são mediados por variáveis individuais e contextuais; por isso exploramos dados sobre o impacto de um conjunto de variáveis capazes de influenciar o curso do luto e determinar as suas consequências. Vimos que a forma como os adultos idosos experienciam a viuvez se encontra associada à forma como experienciam os seus casamentos, isto é à qualidade da relação conjugal. Vimos, também, que as mortes súbitas estão relacionadas com níveis elevados de pensamentos intrusivos, e que os níveis de ansiedade aumentam, seis e dezoito meses após a perda, quando a morte é esperada. Vimos, também, que os valores da depressão são mais elevados junto dos homens viúvos, quando comparados com as mulheres viúvas, quando o tempo de viuvez é superior a 4 anos.

Embora as variáveis que aqui explorámos não esgotem toda a complexidade que é o processamento do luto podem, contudo, contribuir para uma melhor compreensão e esclarecimento das diferenças individuais e da vulnerabilidade de alguns indivíduos para apresentarem lutos complicados. Boerner e Schulz (2009) por exemplo, identificaram os aspectos que podem estar presentes num luto complicado, como a existência de uma saudade imensa pela pessoa falecida, pensamentos intrusivos e recorrentes acerca da sua ausência, os quais impedem de algum modo a construção de novas relações interpessoais e a participação em actividades recompensadoras. Neste sentido, devemos mostrar alguma cautela quando olharmos para as conclusões das investigações aqui abordadas. É que o luto revela-se um processo complexo e multidimensional, envolvendo um conjunto de elementos físicos, psicológicos e sociais, cuja interacção deve ser tida em consideração na avaliação do impacto das variáveis no processo de luto. Estas variáveis devam ser enquadradas no contexto social, cultural e demográfico de cada indivíduo. E, sobretudo, embora haja estudos que esclarecem o impacto da perda ao longo do tempo faltam-nos, ainda, modelos que esclareçam o luto numa perspectiva de ciclo de vida.

\section{Linhas de Orientação para Futuras Pesquisas}

Um olhar mais atento para as investigações acabadas de resumir leva-nos a perguntar se os adultos idosos de hoje e do futuro continuarão a desempenhar papéis e tarefas associadas ao género uma vez que, actualmente, a dependência emocional e instrumental associada ao género no casamento tende a redefinir-se acompanhando as mudanças sociais. Este facto poderá vir a ser de especial interesse para futuras investigações. A par do conhecimento do impacto de certas variáveis no processamento do luto, importa colocar na agenda científica o conhecimento da evolução dos processos duais de coping com a perda do cônjuge, num momento da vida tão particular e tão complexo como é a idade adulta avançada. Cremos também que o conhecimento da evolução do processo dual de coping será um instrumento clínico e educativo importante no apoio e cuidados a prestar a esta população, às suas famílias, aos profissionais da saúde e à sociedade em geral.

\section{Referências}

Bennet, K. M. (1997). Widowhood in Elderly Women: The medium-and long-term effects on mental and physical health. Mortality, 2(2), 137-149.

Bennet, K. M. (1998). Longitudinal changes in mental and physical health among Elderly, recently widowed Men. Mortality, 3(3), 265-274.

Bennet, K. M. (2005). Psychological wellbeing in later life: The longitudinal effects of marriage, widowhood and marital status change. International Journal of Geriatric Psychiatry, 20, 280-284. doi:10.1002/gps. 1280

Boerner, K., \& Shulz, R. (2009). Caregiving, bereavement and complicated grief. Bereave Care, 28, 10-13. doi: 10.1080/ 02682620903355382

Bonanno, G., \& Kaltman, S. (1999). Toward an integrative perspective on bereavement. Psychological Bulletin, 6 , $760-776$. 
Silva, M. D. F. \& Ferreira-Alves, J. (2012). O Luto em Adultos Idosos: Natureza do Desafio Individual e das Variáveis Contextuais em Diferentes Modelos.

Bowlby, J. (1980). Loss: Sadness and depression: Vol. 3. New York: Basic Books.

Bradley, M. J., \& Cafferty, P. T. (2001). Attachment among older adults: Current issues and directions for future research. Attachment \& Human Development, 3, 200-221. doi: 10.1080/ 14616730110058016

Canavarro, C. M. (2004). Vinculação, perda e luto: Implicações clínicas. Psychologica, 35, 35-47.

Carr, D. (2006). Good grief: Bounding back from a spouse's death in later life. Contexts, 5, 22-27.

Carr, D., \& Bodnar-Deren, S. (2009). Gender, aging and widowhood. In P. Uhlenberg (Ed.), International handbook of population aging (pp. 705-728). New York: SpringerVerlag. doi:10.1007/978-1-4020-8356-3_32

Carr, D., House, J. S., Kessler, C. R., Nesse, R., Sonnega, J., \& Wortman, C. (2000). Marital quality and psychological adjustment to widowhood among Older Adults: A longitudinal analysis. Journal of Gerontology: Social Sciences, 55B, S197-S207.

Carr, D., House, J. S., Wortman, C., Nesse, R., \& Kessler, C. R. (2001). Psychological adjustment to sudden and anticipated spousal loss Among Older widowed persons. Journal of Gerontology: Social Sciences, 56B, S237-S248.

Carr, D., \&, Utz, R. (2002). Late-life widowhood in the United States: New directions in research and theory. Ageing International, 27, 65-88.

Cavanaugh, J. (1993). Adult development and aging. Pacific Groove, CA: Brooks.

Davison, K. (2002). Gender differences in new partnership choices and constraints for older widows and widowers. Ageing International, 27, 43-60.

Freud, S. (2001). Mourning and melancholia. In J. Strachey (Ed.), The Standard Edition of the Complete Psychological Works of Sigmund Freud: Vol. 14 (pp. 243-258). London: The Hogart Press. (Original work published in 1917)

Hansson, O. R., \& Stroebe, M. S. (2007). Bereavement in late life. Coping, adaptation and developmental Influences. Washington, DC: American Psychological Association.

Hazan, C., \& Shaver, P. (1987). Romantic love conceptualized as an attachment process. Journal of Personality and Social Psychology, 52, 511-524

Johnson, G. J., Vanderwerker, C. L., Bornstein, F. R., Zhang, B., \& Prigerson, G. H. (2006). Development and validation of an instrument for the assessment of dependency among bereaved persons. Journal of Psychopathology and Behavioral Assessment, 28, 263-272. doi: 10.1007/s10862-0059016-3.

Kübler-Ross, E. (1997). On death and dying. New York: Touchstone.

Lee, M.-A., \& Carr, D. (2007). Does the context of spousal loss affect the physical functioning of older widowed persons? A longitudinal analysis. Research on Aging, 29, 457-487. doi: 10.1177/0164027507303171

Lund, A. D., Caserta, S. M., \& Dimond, F. M. (1993). The course of spousal bereavement in later life. In M. S. Stroebe, W. Stroebe, \& H. O. Robert (Eds.), Handbook of bereavement: Theory, research, and intervention (pp. 240-254). Cambridge, MA: Press Syndicate of the University of Cambridge.

Malheiros, F., \& Ferreira-Alves, J. (2010). O efeito das perdas e dos procedimentos éticos na saúde e bem-estar de adultos idosos que vivem em equipamentos sociais. Poster apresentado no VII Simpósio Nacional de investigação em Psicologia, Braga, Portugal
Moss, S. M., Moss, Z. S., \& Hansson, O. R. (2001). Bereavement and Old Age In M. S. Stroebe, O. R. Hansson, W. Stroebe, \& H. Schut (Eds.), Handbook of bereavement and research: Consequences, coping and care (pp. 241-260). Washington, DC: American Psychological Association Press.

Parkes, C. M. (1988). Bereavement as a psychosocial transition: Processes of adaptation to change. Journal of Social Issues, 44, 53-65.

Parkes, C. M. (1993). Bereavement as a psychosocial transition: Processes of adaptation to change. In M. S. Stroebe, W. Stroebe, \& H. O. Robert (Eds.), Handbook of bereavement: Theory, research, and intervention (pp. 91-101). Cambridge, MA: Press Syndicate of the University of Cambridge.

Parkes, C. M. (1998). Coping with loss. Bereavement in adult life. $B M J, 316,856-859$

Peggy, T., \& Kalyani, M. (2001). Participating in the Home. Journal of Aging Studies, 15(2), 127-145.

Rijo, D. (2004). Temas críticos do luto patológico: Diagnóstico, modelos e intervenção terapêutica. Psychologica, 35, 4967.

Russac, J. R., Steighner, S. N., \& Canto, I. A. (2002). Grief work versus continuing bonds: A call for paradigm integration or replacement? Death Studies, 26, 463-478. doi: 10.1080/ 07481180290088266

Sanders, M. C. (1993). Risk factors in bereavement outcome. The course of normal grief. In M. S. Stroebe, W. Stroebe, \& H. O. Robert (Eds.), Handbook of bereavement: Theory, research, and intervention (pp. 255-267). Cambridge, MA: Press Syndicate of the University of Cambridge.

Schaefer, A. J., \& Moos, H. R. (2001). Bereavement experiences and personal growth. In M. S. Stroebe, O. R. Hansson, W. Stroebe, \& H. Schut (Eds.), Handbook of bereavement and research: Consequences, coping and care (pp. 145-167). Washington, DC: American Psychological Association Press.

Stroebe, M. S. (1998). New directions in bereavement research: Exploration of gender differences. Palliative Medicine, 12, 5-12.

Stroebe, M. S., \& Schut, H. (1999). The dual process model of coping with bereavement: Rationale and description. Death Studies, 23, 197-224.

van Grootheest, D. S., Beekman, A. T. F., Broese van Groenou, M. I., \& Deeg, D. J. (1999). Sex differences in depression after widowhood. Do men suffer more? Social Psychiatry and Psychiatric Epidemiology, 34, 391-398.

Ward, L., Mathias, L. J., \& Hitchings, E. S. (2007). Relationships between bereavement and cognitive functioning in Older Adults. Gerontology, 53, 362-372. doi: 10.1159/ 000104787

Worden, W. J. (2002). Grief counselling and grief therapy. A handbook for the mental health practioner. New York: Springer.
Recebido: 23/09/2010

${ }^{a}$ revisão: 24/05/2011

$2^{a}$ revisão: 27/05/2011

Aceite final: $1 \% 06 / 201$ 